\title{
Determination of Chromium Oxidation State in Cultures of Dissimilatory Metal Reducing Bacteria by Electron Energy Loss Spectroscopy
}

\author{
Tyrone L. Daulton, ${ }^{*}$ Brenda J. Little, ${ }^{* *}$ and Kristine Lowe**
}

* Marine Geosciences Division, Naval Research Laboratory, Stennis Space Center, MS 39529, USA

** Oceanography Division, Naval Research Laboratory, Stennis Space Center, MS 39529, USA

Chromium is a redox active $3 d$ transition metal with a wide range $(-2$ to +6$)$ of possible oxidation states; however only $\mathrm{Cr}(\mathrm{III})$ and $\mathrm{Cr}(\mathrm{VI})$ are stable. The valence state of $\mathrm{Cr}$ is important because it controls the geochemistry and toxicity of the element. Hexavalent $\mathrm{Cr}$ species are strong oxidants which act as carcinogens, mutagens, and teratogens in biological systems [1]. The high solubility, bioavailability, and toxicity of $\mathrm{Cr}(\mathrm{VI})$ make it a particular environmental concern. In contrast, $\mathrm{Cr}$ (III) species have low toxicity, in part, because their bioavailability is limited by their low solubility and their tendency to form strong complexes with organics and hydroxo complexes. Hexavalent $\mathrm{Cr}(\mathrm{VI}) \mathrm{can}$ be readily reduced to the trivalent state by $\mathrm{Fe}^{2+}, \mathrm{S}^{2-}$, organic compounds, wetland plants, and several species of microorganisms [2]. The associated mechanisms of $\mathrm{Cr}$ (VI) reduction are technologically and biologically important because they convert a toxic, mobile element into a less toxic, immobile

form. Furthermore, the geochemical and microbiological processes of $\mathrm{Cr}$ oxidation/reduction drive both precipitation/dissolution and ion adsorption/desorption reactions in the environment. The study of these reactions has been hindered by the lack of an analytical technique that can determine the oxidation state of $\mathrm{Cr}$ at high spatial resolution. For instance, little is known about unstable redox intermediates with intermediate valence states between the valances of the stable end members of the oxidation/reduction process.

Electron energy loss spectroscopy (EELS) techniques have been developed to determine oxidation state of $3 d$ and $4 d$ transition metals at high spatial resolution [3-5]. However, only limited EELS studies of a few $\mathrm{Cr}$ oxidation states have been performed, and with the exception of our work [6], none have been applied to biological systems. We have previously reported the application of several EELS techniques in combination for the study of $\mathrm{Cr}(\mathrm{VI})$ reduction by the bacterium Shewanella oneidensis using conventional- and in-situ environmental cell (EC)- transmission electron microscopy (TEM) [6]. Shewanella oneidensis ( $S$. oneidensis) is a gram-negative, facultative bacterium capable of respiring aerobically and anaerobically using a variety of compounds/metals as terminal electron acceptors including $\mathrm{Cr}(\mathrm{VI})$. In our previous work, a limited number of standards were examined. Here we report detailed EELS measurements of $\mathrm{L}_{3} / \mathrm{L}_{2}$ integrated-peak intensity ratios and $\mathrm{L}_{3}$ peak positions for a greatly expanded range of chromium compounds, including additional oxidation states. Previously analyzed $\mathrm{Cr}$ standards are also reanalyzed, and we report less relative scatter in their data than in the original study [6], a consequence of repairs and upgrades to the energy loss spectrometer. Further, we report results of EELS studies of $\mathrm{Cr}$ reduction in anaerobic cultures of $S$. oneidensis.

\section{References:}

[1] M. Cieslak-Golonka, Polyhedron 15 (1995) 3667-3689.

[2] S. Fendorf, B. W. Wielinga, C. M. Hansel, Internat. Geol. Rev. 42 (2000) 691-701

[3] K. M. Krishnan, Ultramicroscopy 32 (1990) 309-311.

[4] J. H. Paterson, O. L. Krivanek, Ultramicroscopy 32 (1990) 319-325.

[5] P. A. van Aken, B. Liebscher, V.J. Styrsa, Phys. Chem. Min. 25 (1998) 323-327.

[6] T. L. Daulton, B. J. Little, K. Lowe, J. Jones-Meehan, Microsc. Microanal. 7, (2001) 470-485.

[7] Work supported under ONR/NRL program element $0602236 \mathrm{~N}$. 


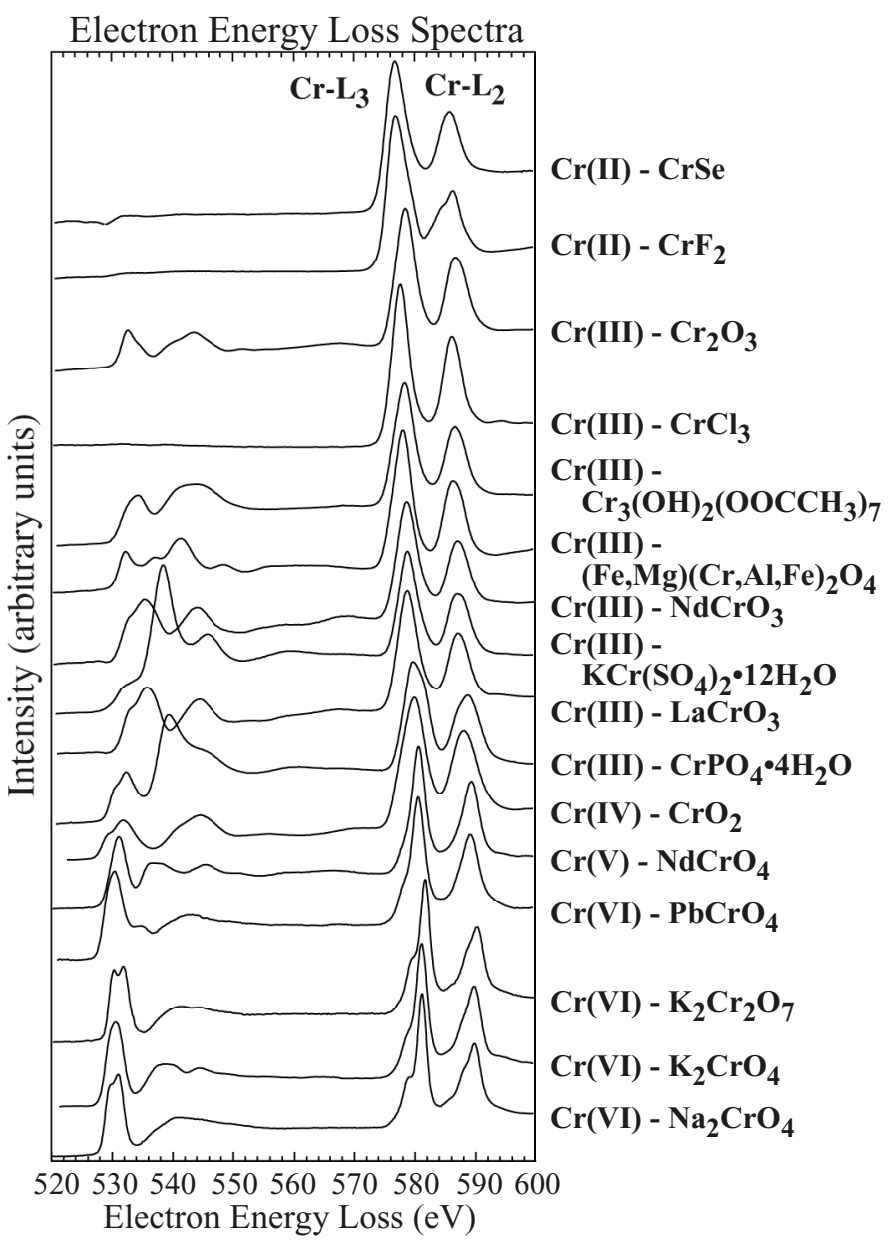

Figure 1. Comparison of core-loss EELS spectra of $\mathrm{Cr}$ oxidation state standards. Spectra were normalized to the intensity of the $\mathrm{L}_{3}$ peak and offset from one another. They represent the sum of 25 individual spectra. The spectrometer was calibrated to the edge maxima of $\mathrm{Ni}-\mathrm{L}_{3}(\mathrm{NiO})=855.00 \pm 0.03$ $\mathrm{eV}$. Instrument details are given in Ref. 6 .

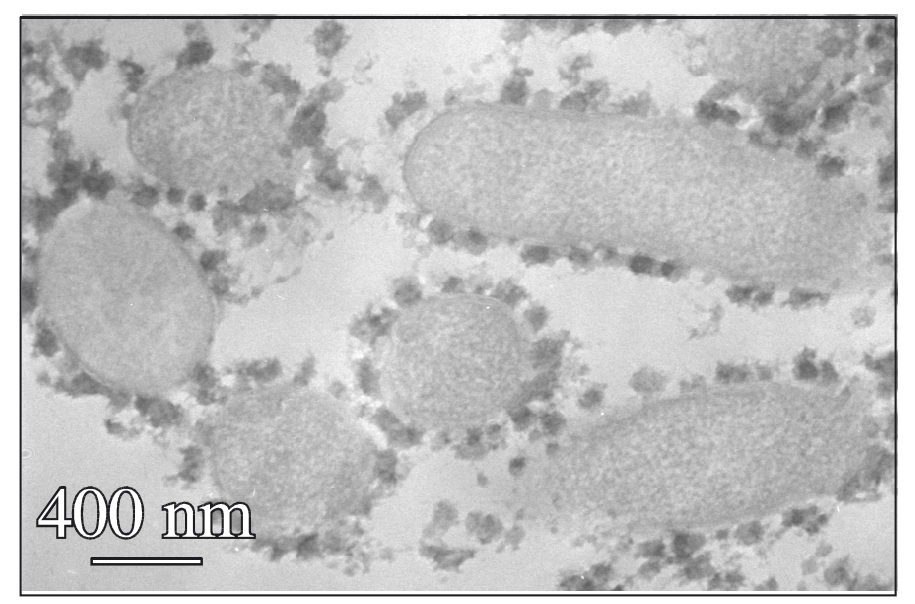

Figure 2. Encrusted S. oneidensis in cross-section.

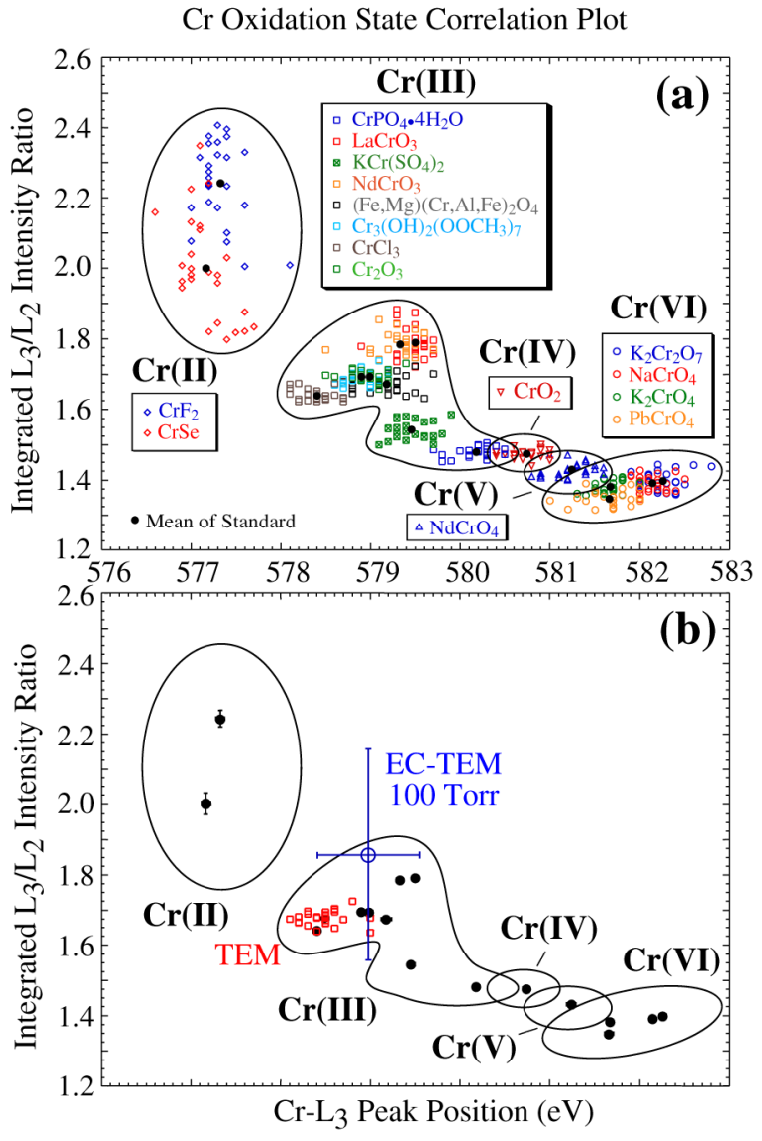

Figure 3. Correlation between $\mathrm{L}_{3} / \mathrm{L}_{2}$ integrated-peak intensity ratios and $\mathrm{L}_{3}$ peak positions for $\mathrm{Cr}$ oxidation-state standards (a). For analysis details see Ref. 6. Solid data points represent the mean of the data for a particular standard. Different $\mathrm{Cr}$ oxidation states fall within well-separated regions in the correlation plot. Within a given oxidation state, spectra for the individual standards fall within separate groupings reflecting possible differences in atom coordination, spin-orbit interactions, crystal field splitting, atomic coulomb repulsion, and exchange effects between the compounds. These factors also influence $\mathrm{L}_{2,3}$ fine structure and must be considered to properly interpret absorption edges. The correlation plot represents a map of the possible range in fine structure (including influences from factors other than valence state) that a particular $\mathrm{Cr}$ oxidation state can display. The mean oxidation state of an unknown can be determined by plotting its $\mathrm{L}_{3}$ peak position and $\mathrm{L}_{3} / \mathrm{L}_{2}$ integrated-peak intensity ratio in a correlation plot of oxidation state standards as shown for $\mathrm{Cr}$ precipitates encrusting S. oneidensis in (b). 